Lymphology 52 (2019) 187-193

\title{
EFFECT OF PREGNANCY ON LOWER LIMIB LYMPHEDEMA IN PATIENTS TREATED WITH MULTISITE LYMPHATICOVENULAR ANASTOMOSES (MLVAS)
}

I. Koshima, Sy. Yoshida, S. Nagamatsu, K. Yokota, H. Mizuta, M. Harima, K. Tashiro, Sh. Yamashita, I. Ratanshi, T. Eldahshoury

International Center for Lymphedema (IK,SyY) and Plastic and Reconstructive Surgery (SN,KY), Hiroshima University Hospital, Hiroshima City, Japan; Department of Plastic and Reconstructive Surgery (HM,MH,KT,ShY), Graduate School of Medicine, The University of Tokyo, Japan; Plastic \& Reconstructive Surgery Service, Department of Surgery (IR), Memorial Sloan Kettering Cancer Center, New York, New York, USA; and Department of Plastic and Reconstructive Surgery (TE), Salisbury District Hospital, Salisbury United Kingdom

\section{ABSTRACT}

Lymphaticovenular anastomosis (LVA) using supermicrosurgical techniques is effective for treating and preventing progression of lymphedema. We analyzed the influence of pregnancy on LVA in five patients from a total 2179 LVA cases. Previous studies offer conflicting reports on whether pregnancy worsens pre-existing lymphedema. This is the first report on the influence of pregnancy on lower limb lymphedema previously treated by multisite LVA (mLVA). Five patients with primary $(n=4)$ and secondary $(n=1)$ lower leg lymphedema were analyzed for this study. Patient age ranged from 18 to 31 (average 22.6) years old with 4 right and 1 left extremities involved. Duration of symptoms ranged from one to 19 (average 7.4) years and the periods of compression therapy were from 1 to 19 years (6.6 years). Four patients had single pregnancies and one patient was multiparous with 3 pregnancies. Final follow-up ranged from 5.8 to 18 years (average 8.9 years) after the primary $m L V A$. All patients had normal pregnancy, birth, and no serious complications after surgeries. Following pregnancy three patients had complete functional recovery (limb volume reduction and no compression requirement), one with function- al improvement (limb volume reduction but required compression), and one with no change in symptoms (not worse and continued need for compression). There were no occurrences of infection following pregnancy. Based on this case series, it is suggested that pregnancy does not worsen the pre-existing lymphedema in patients who had previously undergone mLVA. Further studies with larger number of patients are needed to confirm these results.

Keywords: lymphedema, pregnancy, lower limb, primary lymphedema, lymphaticovenous anastomosis, lymphaticovenular anastomosis, LVA, multisite LVA, mLVA, microsurgery, supermicrosurgery, cellulitis, physiotherapy, compression therapy

Lymphedema is a progressive disease resulting in chronic swelling and eventual deposition of fibroadipose tissue in affected regions of the body. Congenital or developmental dysfunction in lymphatic channels are causes of primary lymphedema, which can present at birth, adolescence, or adulthood. Patients may also develop lymphedema secondary to infection, trauma, tumor extirpation, or lymph node dissection. For decades, non-operative modalities of limb elevation, compression, 
manual decongestive therapy, and weight loss have been the mainstay of lymphedema treatment. Adjunctive surgical strategies have sought to remove accumulated fibroadipose tissue in limbs recalcitrant to conservative measures by way of liposuction or formal excision. Advances in micro- and supermicrosurgical techniques offer promise to improve lymphatic drainage by shunting accumulated lymph into the venous system.

Microvascular lymphaticovenular anastomosis (LVA) was first proposed by O'Brien and colleagues for obstructive lymphedema (1-3). Long-term results reported by $\mathrm{O}^{\prime} \mathrm{Brien}$ and colleagues, along with other groups, demonstrated volume reduction or subjective benefit in a large subset of patients undergoing LVA. Yet, reproducibility of these results was inconsistent limiting widespread adoption among microsurgeons (4-6). It was the lead authors' hypothesis that the major limiting factor influencing outcomes was surgical technique, where more precise instrumentation, visualization, and handling strategies were required to better manage vascular anastomoses on a sub-millimeter scale.

To address this limitation, the lead authors of this article developed 'supermicrosurgery' techniques which enabled precise microanastomosis of vessels 0.3 to $0.8 \mathrm{~mm}$ in caliber (7-10). This required the development of superfine microsurgical instruments and smaller diameter sutures (here, 12-0 nylon with a 50 micrometer diameter needle) to carry out six to eight water tight sutures between lymphatic channels and nearby venules of the subdermal plexus. Performing multisite LVA (mLVA) using supermicrosurgery techniques has demonstrated improved results. Since 1990, the authors have performed LVA in 2179 patients of which 1900 involved the lower extremity and 279 cases involved the upper extremity mostly under local anesthesia. Remarkable improvement to complete recovery was obtained in mild to moderate cases (7-10).

Women of childbearing age may present with primary lymphedema or develop the disease from any of the aforementioned caus- es. Pregnancy has been shown to not worsen lymphedema features in most patients $(11,12)$. However, the pregnancy state is associated with an increase in overall fluid volume and intravascular pressures of the superficial venous system (13). Given that successful LVA techniques depend on the superficial venous system to provide an avenue for lymphatic outflow, it is unclear if higher venous pressures or pregnancy-related volumetric changes influence the long-term results in patients who have demonstrated a successful response to LVA. There are currently no reports demonstrating the effect of pregnancy on lymphedema in patients who have been previously treated with LVA. The purpose of this study is to review our experience with this unique patient population.

\section{MATERIALS AND METHODS}

We retrospectively reviewed all adult patients (age > 18 years) who underwent elective surgical treatment for lymphedema with LVA by the senior author (I.K.) from 1999 to 2017 and who had a subsequent pregnancy.

\section{Operative Technique}

Patients who presented with mild to severe lymphedema and were compliant with compressive garments and decongestive therapy underwent operative treatment with mLVA. All surgeries were performed under local anesthesia on adult patients. Two or more $3-\mathrm{cm}$ long incisions were used for exposure. Lymphatics and subdermal venules (each ranging from 0.2 to $0.5 \mathrm{~mm}$ in diameter) were identified under loupe magnification or using an operating microscope. Affected lymphatics were often dilated or sclerotic. Where dye staining was unable to identify lymphatic channels, additional incisions proximally (above knee) or distally (e.g., foot dorsum) were made. Subdermal venules were often identified anywhere immediately below the dermis or deeper, often adjacent to lymphatics. Lymphatic channels suitable for anastomosis were chosen based 
on strong drainage function, which can be assessed when transecting the channel under an operating microscope. Small subdermal venules, ideally $0.5 \mathrm{~mm}$ or less in caliber are most suitable for anastomosis with lymphatic channels as larger cutaneous veins may have higher intravascular pressure, limiting outflow. We do not use a vascular clamp. End-to-end anastomoses were performed with a 12-0 nylon suture on 50 micrometer needle and superfine needle holder under high magnification of 20-30X (Fig. 1). Although often completed under 3 hours, patients (particularly those with previous pelvic radiation) required urinary catheterization.

Post-operatively, no bandage was applied and patients were encouraged to ambulate immediately. All patients were kept in hospital for 1 week to receive Prostaglandin E1, a vascular dilating agent. Two weeks later, patients were fitted for elastic compression stockings to be worn for at least 6 months. (Our weaning protocol for compression garments: in a mild garment at 1 week, and then a stronger compression garment at 3-4 weeks, no physiotherapy. Antibiotics were given for 1week. No liposuction prior to becoming pregnant)

\section{Clinical Outcome Evaluation}

In patients who had undergone previous mLVA, pre- and postpartum concerns included subjective worsening of lymphedema, requirement of compression, and occurrence of post-operative infection requiring hospital admission.

Lymphedema evaluation was based on patient subjective concerns and periodical morphometric analysis using photographic assessment taken at every three months.

\section{RESULTS}

Five patients were identified from a total of $\mathbf{1 9 0 0}$ lower extremity lymphedema cases who underwent LVA surgery and who subsequently became pregnant post-operatively. In one case, the patient received an additional procedure - a vascularized lymphatic channel transfer from the lateral thoracic region after undergoing an LVA previously. There were 4 cases of primary lymphedema and one case of secondary lymphedema due to trauma. There were no patients with a history of familial lymphedema. LVA was performed in patients aged 18 to 31 years (average 22.6 years) with a total of 5 unilateral lower extremities (4 right: 1 left). Duration of lymphedema ranged from 1 to 19 years (average 7.4 years). All patients were compliant with compression for at least 1 year prior to undergoing LVA (range 1-19 years, average 6.6 years). Two of these patients had occurrences of infection requiring hospital admission for intravenous antibiotics involving the lymphedematous extremity prior to undergoing LVA.

Number of LVA performed per patient ranged from 1 to 3 (average 1.4) and were accomplished in a 3 hour setting. At final follow-up prior to pregnancy, 3 patients had an excellent result with complete resolution of edema and did not require compression and 1 patient had moderate improvement with reduced swelling and no compression requirement. One patient did not demonstrate any improvement of symptoms and was maintained on compression.

All patients had a normal pregnancy, birth and no serious complications associated with childbearing or their LVA surgery prior. Of the included patients, four had 1 pregnancy and one patient had 3 pregnancies. Functional outcomes remained unchanged pre- and post-pregnancy.

A summary of patients included in this case review is provided in Table.

\section{Representative Case Examples}

Case 2: LVA for primary lymphedema and a single pregnancy (Figs. 1A and $1 B$ )

A 21-year-old woman suffered from primary lymphedema of the left leg complicated by an episode of cellulitis. Physiotherapy and compression was administered for three years 


\begin{tabular}{|c|c|c|c|c|c|c|c|}
\hline \multicolumn{8}{|c|}{$\begin{array}{c}\text { TABLE } \\
\text { Summary of patients }\end{array}$} \\
\hline Case & Age & Side & History & $\begin{array}{l}\text { Frequency } \\
\text { of surgery }\end{array}$ & Pregnancy & Follow & Final results \\
\hline 1 & $31 y$ & $\mathbf{R}$ & L20yEC1yP- & LVA2x & $1 x$ & $18 y$ & no compression \\
\hline 2 & $21 y$ & $\mathbf{L}$ & LpE3yC1yP1 & LVA1x & $1 x$ & $8 y$ & no compression \\
\hline 3 & 18 & $\mathbf{R}$ & LpEC6yP2 & LVA2x & $1 x$ & $8 y$ & no compression \\
\hline 4 & 24 & $\mathbf{R}$ & LpE7yC6yP- & LVA1x & $3 x$ & $5 y 10 m$ & no change \\
\hline 5 & 19 & $\mathbf{R}$ & congL19yEC19yP- & LVA1x & $1 x$ & & excellent \\
\hline average & $22.6 \mathrm{y}$ & & E7.4y C6.6y & $1.4 x$ & $1.4 x$ & $8.9 y$ & \\
\hline
\end{tabular}

(R=right; L=left; L20y= latency period of 20 years after skin graft; $\mathrm{Lp}=$ primary lymphedema; cong=congenital lymphedema; $E=$ duration of lymphedema; $C=$ duration of compression; $P=$ frequency of deep cellulitis; $L V A=$ lymphaticovenular anastomosis; LVA $x$ 1=one LVA performed; no compression=no need for compression; pregnancy=frequency of pregnancy and birth).

An "excellent result" means continued need for compression and is different from "no compression required"; "no change" refers to patients who did not have any change in their lymphedema and still require compression.

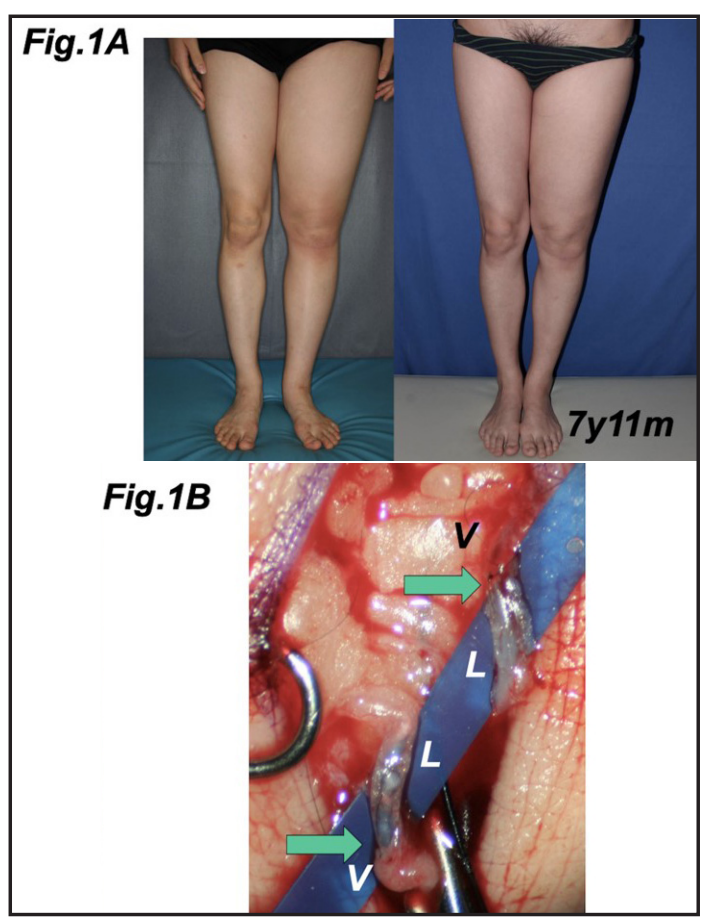

Fig. 1. Case 2 with primary lymphedema for three years and one episode of infection. 1A (left): Preoperative condition. Compression was not able to stop progression of edema or infection. 1A (right) Patient demonstrates functional recovery with no compression at seven years and 11months after surgery and two years after one birth. 1B Operative photograph at the left ankle demonstrates multiple lymphaticovenular anastomosis (LVA) under operating microscope. Lymphatics $(L)$ are dilated and translucent. $V$ indicates subdermal venules and the arrowheads show the anastomoses. without improvement. Six LVA under local anesthesia were performed along the medial aspect of the left leg. Postoperatively, the patient's lymphedema improved, and by four years post she no longer required therapy or compression garments. Six years after surgery, she had an uncomplicated pregnancy without any worsening of the involved extremity. At final follow-up seven years and 11months after the LVA (two years post-pregnancy), circumference was decreased after pregnancy and she did not require any additional compressive or physiotherapy.

\section{Case 4: LVA for primary lymphedema and multiple pregnancies (Fig. 2)}

A 24-year-old woman suffered from primary lymphedema of the right leg whose onset presented at age 17 . She was treated with continued physiotherapy and compression for six years. Six LVA were performed along the medial aspect of the right leg. While her limb did not decrease in volume, it remained responsive to compressive therapy. Three years and seven months after surgery, she had a child and her lymphedema remained the same size as before pregnancy. She still needed compression. At four years and seven months after surgery, she had a second child and edema of the right leg worsened despite constant compression. Six years post-operatively, she had her third child. 


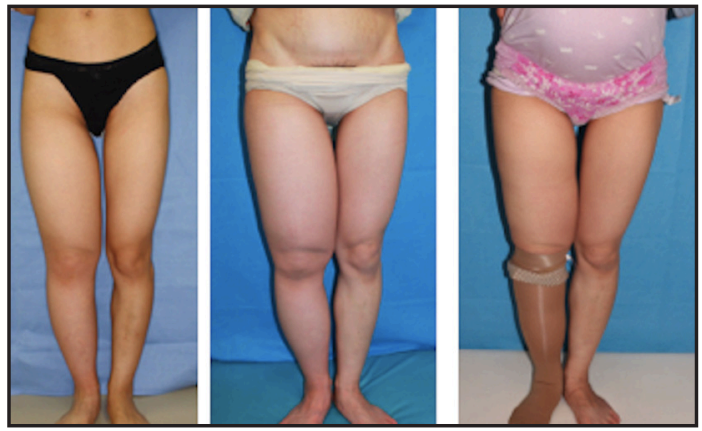

Fig. 2. Case 4 with primary lymphedema for seven years. (left) Preoperative condition. (center) Patient image following two births and four years and seven months after surgery. Fat hypertrophy in the thigh is due to increased body weight. (right) Nine months after the third pregnancy and five years and 10 months after LVA surgery. Edema was maintained with compression.

Over successive pregnancies, she experienced generalized weight gain resulting in some accumulated adipose tissue in her limb, but the consistency was similar to her other limbs and was not fibrofatty. Overall, her lymphedema remained constant throughout this course and did not worsen.

Case 5: LVA for congenital lymphedema and a single pregnancy (Fig. 3)

A 19-year-old woman with congenital lymphedema of the right leg was treated with continued physiotherapy and compression for 19 years. Four LVA were performed along the medial aspect of the right leg. After surgery, her limb decreased in volume. Two years and one month after surgery, without any worsening of the involved extremity, she had a child and her lymphedema continued to demonstrate the same size as before pregnancy. By five years after surgery (three years post-pregnancy), the circumference remained the same as before pregnancy, but required compressive therapy.

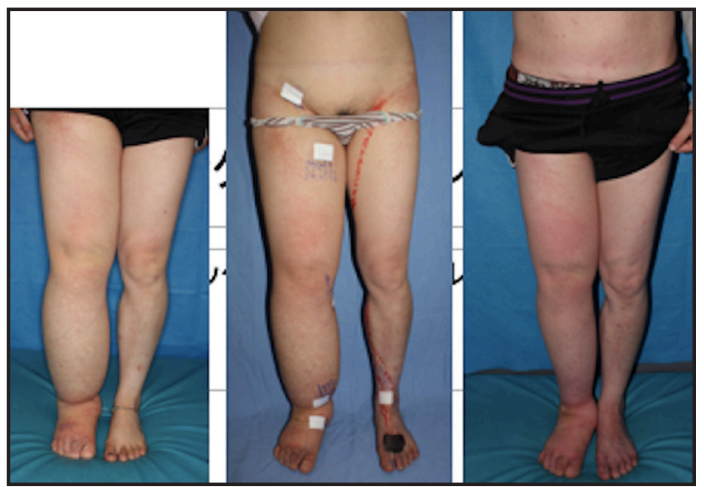

Fig. 3. Case 5 with congenital lymphedema for 19 years. (left) Preoperative condition. Compression did not stop progression of edema. (center) Patient immediately after surgery consisting of four LVA for the right leg and one prophylactic for left leg (right). $\mathrm{Pa}$ tient three years after birth and five years after LVA operation. Dramatic improvement using the same compression as before the surgery.

\section{DISCUSSION}

Young women of childbearing age can be affected by lymphedema. There are a limited number of studies available describing the interaction of the pregnancy with lymphedema. This is likely due to the relatively rare incidence of primary or secondary lymphedema in young patients in industrialized nations and limited access to therapy and clinical follow-up in the developing world where lymphedema is more likely to impact women of childbearing age.

In their short series of 15 patients, Brunner and Lachat observed that although lymphedema may vary in first and second trimesters, it returns to an irreversible state following pregnancy and can worsen with multiparity (11). Vignes and colleagues' study was more encompassing and described the course of 49 women at a single center over 4 years (12). These patients were mainly treated with compression and lymphedema therapy. In contrast to Brunner's results, only $10 \%$ had subjective complaints of exacerbated disease, while $50 \%$ of women experienced longer lasting, albeit transient, increase of limb edema. Of the four patients who were multiparous and experi- 
enced worsening lymphedema after the first pregnancy, lymphedema was further worsened after a second pregnancy (12). While one can infer early onset lymphedema may not worsen with lymphedema, limited patient numbers and heterogeneity of causative and patient factors limit generalizability. They also do not offer guidance as to which subset of lymphedema patients (e.g., primary vs. secondary, acquired vs. inherited, etc.) may be at greater risk of disease progression.

We have previously shown the usefulness of LVA for lower limb lymphedema from both primary and secondary causes. Outcomes can range from reduction of concerning symptoms and complete independence from compression therapy to absent improvement in some. Despite having completed this technique in over 2100 patients, we identified only 5 patients who became subsequently pregnant. Low numbers are reflective of both the rare incidence of lymphedema among younger patients in Japan and other developed countries and the few centers worldwide with capacity to offer physiologic procedures for lymphedema, such as LVA. All but one patient (case 4) demonstrated complete or near-complete functional recovery with improvement of symptoms post-pregnancy except for one patient with primary lymphedema who required continued compression therapy. Patients observed in this study also had a relatively longer follow-up (8.6 years after surgery) compared to other studies reported in the literature.

The mechanism of lymphatic drainage is facilitated by active contraction of smooth muscle cells within tunica media of collecting lymph channels, which have arterioles and venules on the outer surface of lymphatics (i.e., a vasa lymphorum). Based on the lead author's (I.K.) electron microscopic study, even in primary edema, early stage lymphedema is characterized by loss of these feeding vessels, whereas later stages exhibit degeneration of smooth muscle cells and subsequent disorganized smooth muscle cell regeneration (7). Recurrent episodes of lymphangitis produce sclerosis, obstruction, and fibrotic change, which completely damage the drainage function. Irradiation, obesity, and infection are extrinsic factors that can seriously exacerbate lymphedema. We have observed that even in mild cases of lymphedema, loss of feeding vessels and lymph channel sclerosis can be identified.

Our series suggests that patients who showed improvement due to LVA are likely to maintain their functional gains following pregnancy. These patients may or may not require the use of additional compression or physiotherapy during pregnancy. However, given the limited number of patients observed in this series, we cannot determine the influence of other contributors of lymphedema on outcomes, such as primary lymphedema or secondary lymphedema along with duration of symptoms. Although the studies by Brunner and Lachat, and Vignes and colleagues showed a subset of patients whose lymphedema worsened during pregnancy $(11,12)$, conclusions cannot be drawn as of yet if LVA can reduce the effect of pregnancy in these patients. Further studies are needed to better evaluate this population. The primary fear of patients afflicted with lymphedema who show improvement is symptom recurrence. This study may be of benefit in helping advise patients on expected outcomes following mLVA and pregnancy.

\section{CONFLICT OF INTEREST AND DISCLO- SURE}

All authors declare no competing financial interests exist.

\section{REFERENCES}

1. O'Brien, BM, LA Chait, PJ Hurwitz: Microlymphatic surgery. Orthoped. Clin. N. Amer. 8 (1977), 405-424.

2. O'Brien, BM, PJ Sykes, GN Threlfall, et al: Micro-lymphaticovenous anastomoses for obstructive lymphedema. Plast. Reconstr. Surg. 60 (1977), 197-211. 
3. O'Brien, BM, CG Mellow, RK Khazanchi, et al: Long-term results after microlymphaticovenous anastomoses for the treatment of obstructive lymphedema. Plast. Reconstr. Surg. 85 (1990), 562-572.

4. Huang, GK, RQ Hu, ZZ Liu, et al: Microlymphaticovenous anastomosis in the treatment of lower limb obstructive lymphedema: Analysis of 91 cases. Plast. Reconstr. Surg. 76 (1985), 671-677.

5. Campisi, C: Use of autologous interposition vein graft in management of lymphedema: Preliminary experimental and clinical observations. Lymphology 24 (1991), 71-76.

6. Campisi, C, F Boccardo, A Zilli, et al: Longterm results after lymphatic-venous anastomoses for the treatment of obstructive lymphedema. Microsurg. 21 (2001), 135-139.

7. Koshima, I, S Kawada, T Moriguchi, et al: Ultrastructural observation of lymphatic vessels in lymphedema in human extremities. Plast. Reconstr. Surg. 97 (1996), 397-405.

8. Koshima, I, K Inagawa, K Urushibara, et al: Supermicrosurgical lymphaticovenular anastomosis for the treatment of lymphedema in the upper extremities. Reconstr. Microsurg. 16 (2000), 437-442.

9. Koshima, I, Y Nanba, T Tsutsui, et al: Longterm follow-up after lymphaticovenular anastomosis for lymphedema in the legs. $\mathrm{J}$. Reconstr. Microsurg. 19 (2003), 209-215.
10. Koshima, I, M Narushima, M Mihara, et al: Lymphadiposal flaps and lymphaticovenular anastomoses for severe leg edema: Functional reconstruction for lymph drainage system. J. Reconstr. Microsurg. 32 (2016), 50-55.

11. Brunner, U, M Lachat: Primary lymphedema and pregnancy. Phlebologie. 42 (1989), 481484.

12. Vignes, $S, M$ Arrault, R Porcher: Subjective assessment of pregnancy impact on primary lower limb lymphedema. Angiology 61 (2010), 222-225.

13. Sparey, C, N Haddad, G Sissons, et al: The effect of pregnancy on lower limb venous system of women with varicose veins. Eur. J. Endovasc. Surg. 18 (1999), 294-299.

\section{Isao Koshima, MD \\ Professor and Chief \\ International Center for Lymphedema \\ Hiroshima University Hospital \\ 1-2-3, Kasumi, Minami-ku \\ Hiroshima City, 734-8551 Japan \\ Email: koushimaipla@gmail.com \\ Phone and Fax: +81-82-257-1985}

